\title{
COMPUTER-AIDED MODEL OF COLONIC PROPULSIVE ACTIVITY
}

\author{
O. AL QATRAWI \& R. N. MIFTAHOF \\ Arabian Gulf University, Manama, Kingdom of Bahrain.
}

\begin{abstract}
A biomechanical model and mathematical formulation of the problem of propulsion of a solid nondeformable pellet by an isolated segment of the colon are presented. The organ is modeled as a soft orthotropic cylindrical biological shell. Its wall is reinforced by transversely isotropic muscle fibers of orthogonal type of weaving embedded in a connective tissue stroma. The mechanical properties of the wall are assumed to be nonlinear, deformations are finite. The longitudinal and circular smooth muscle syncitia possesses electrical properties and are under control of a pacemaker, which is represented by the interstitial cell of Cajal. The model describes the dynamics of the generation and propagation of the mechanical waves of contraction-relaxation along the surface of the bioshell and propulsion of the pellet. The governing system of equations has been solved numerically. The combined finite-difference and finite-element method has been used. The results of numerical experiments demonstrate that pendular movements alone provide a normal transit, without mixing though, of the bolus. Non-propagating segmental contractions show small amplitude librations of the pellet without its visible propulsion. Only the coordinated activity of the longitudinal and circular smooth muscle layers in a form of the peristaltic reflex provides physiologically significant simultaneous propulsion and mixing of the intraluminal content.
\end{abstract}

Keywords: Bolus, colon, Computational biology, gastrointestinal tract

\section{INTRODUCTION}

The primary functions of the large intestine (colon) are to store, process and expel fecal mass residues. These require sustained motor activity - the generation of migrating myoelectrical complexes (MMC) that mix and propel the content. Patterns of MMCs produced by the colon under normal physiological conditions are associated with non-propagating and high amplitude propagating mass movements. They occur regularly and assist to push the bulky content ahead of them. The disparity between mechanical and propulsive activities caused by anatomical and/or neuropathological changes in the colon, e.g. Hirschpurg' disease, irritable bowel syndrome, result in idiopathic/slow transit constipation, obstructed defecation or diarrhea. The severity and diversity of clinical symptomatology, relative inaccessibility and the complexities posed by the presence of solid or semi-solid fecal masses, along with the heterogeneity of in vitro experimental findings, makes it difficult to unravel underlying pathophysiological mechanisms and thus design effective treatments.

\section{AIM}

The aim of the study is to investigate numerically the propulsion of the intraluminal content (bolus) and to assess the dynamics of stress-strain distribution, changes in the shape and configuration of the colon.

\section{METHODS}

Below are the basic assumptions that have been used in model construction [1-3]:

1. The colon is a soft orthotropic tube of cylindrical shape. The wall of the colon has two muscle layers and a network of connective tissue. The outer layer contains muscle fibers 
that are organized in the longitudinal direction of the organ while muscle fibers of the inner layer are arranged in a direction that is specified as orthogonal and circular.

2. The rigid sphere of the radius, $\mathrm{R}$, is included within the tubular segment of length, 1 , and radius, $\mathrm{r} 0$, where isometric contractions take place. Throughout the mechanical reactions, the intraluminal pressure, $\mathrm{p}$, varies in accordance with the adiabatic law.

3. The contact forces that takes place between the two parties: the wall and rigid sphere are assumed to be orthogonal to the sphere surface. Dryness and viscous friction are subject of the motion of the bolus.

4. Muscle layers contract in an independent way with generation of active forces (Ta). The first contractions begin with the longitudinal muscle layer.

5. It is assumed that the muscle layers are syncytia that possess cable electrical properties. As for the electrical properties, the longitudinal layer is characterized to be anisotropic while the circular layer is isotropic.

6. The pacemaker cell that is located at the left boundary controls the mechanical activity of the tube, and hereby generates an excitatory stimulus of given intensity.

7. The dynamics of the colon segment is given by:

$$
\gamma_{0} \frac{d V}{d t}=\left(T_{1} e_{i j} \sqrt{g_{22}}\right) s_{1}+\left(T_{2} e_{i j} \sqrt{g_{11}}\right) s_{2}+p \sqrt{g_{j}},
$$

where:

$$
\mathrm{T}_{1(2)}=\mathrm{k} \frac{\partial \varepsilon_{\mathrm{c}(1)}}{\partial \mathrm{t}}+\varphi_{\mathrm{c}(1)} \mathrm{T}^{\mathrm{a}}\left(\lambda_{\mathrm{c}(1)} \mathrm{T}^{\mathrm{p}}\left(\lambda_{\mathrm{c}}, \lambda_{1}\right)\right.
$$

Here $-\gamma_{0}$ the linear density of a biomaterial in an undeformed sate; $\lambda_{c}, \lambda_{1}-$ the rate of elongation (here after the subscripts 1 and $\mathrm{c}$ refer to the longitudinal and circular muscle layers, respectively; - the direction cosines of the $\varepsilon_{\mathrm{c}, 1}=\lambda_{\mathrm{c}, 1}+1$; $\mathrm{e}_{\mathrm{ji}}$ outward normal $\mathrm{n}_{\mathrm{j}}$, to the surface with respect to the cylindrical $\mathrm{j}$ - axis $(I=1,2 ; j=r, s, z) ; g_{i j}, g-$ the components and determinant of the fundamental tensors; $\mathrm{V}\left(\mathrm{V}_{\mathbf{r}}, \mathrm{V}_{\mathbf{s}}, \mathrm{V}_{\mathbf{z}}\right)$ - the velocity vector and its radial circumferential and longitudinal components; $\mathrm{T}_{1,2}-$ the components of the tensor of membrane forces; $\mathrm{T}^{\mathrm{p}}, \mathrm{T}^{\mathrm{a}}-$ the passive and active components of the total force $\left(\mathrm{T}_{\mathrm{c}, \mathrm{l}}\right) ; \mathrm{k}$ - the rheological parameter; $\mathrm{p}$ - intraluminal pressure; $\mathrm{s}_{1}, \mathrm{~s}_{2}$ the Langragian coordinates of the bioshell.

The passive $\mathrm{T}_{c, 1}^{\mathrm{p}}$ components are derived from:

$$
\mathrm{T}_{(\mathrm{c}, 1)}^{\mathrm{p}}=\frac{\partial \gamma_{\mathrm{o}} \mathrm{W}}{\partial\left(\lambda_{(\mathrm{c}, 1)}-1\right)},
$$

where $\mathrm{W}$ - the strain energy density function has the form:

$$
\begin{aligned}
\gamma_{0} \mathrm{~W}= & \frac{1}{2}\left[\mathrm{c}_{1}\left(\lambda_{1}-1\right)^{2}+2_{\mathrm{c}_{3}}\left(\lambda_{1}-1\right)\left(\lambda_{\mathrm{c}}-1\right)^{2}+\mathrm{c}_{2}\left(\lambda_{\mathrm{c}}-1\right)^{2}\right]+ \\
& +\mathrm{c}_{10} \exp \left[\mathrm{c}_{4}\left(\lambda_{1}-1\right)^{2}+\mathrm{c}_{5}\left(\lambda_{\mathrm{c}}-1\right)^{2}+2_{\mathrm{c}_{6}}\left(\lambda_{1}-1\right)\left(\lambda_{\mathrm{c}}-1\right)\right] .
\end{aligned}
$$

As for the active components $\left(\mathrm{T}_{\mathrm{c}, 1}^{\mathrm{a}}\right)$ components we have:

$$
\mathrm{T}_{(\mathrm{c}, 1)}^{\mathrm{a}}=\mathrm{c}_{7(\mathrm{c}, 1)} \lambda_{(\mathrm{c}, 1)}^{2}+\mathrm{c}_{8(\mathrm{c}, 1)} \lambda_{(\mathrm{c}, 1)}+\mathrm{c}_{9(\mathrm{c}, 1)} .
$$

The mechanical constant of a biocomposite are $c_{1-10}$. 
8. The dynamics of propagation of the electrical waves of depolarization along the anisotropic longitudinal muscle layer is $\left(\varphi_{1}\right)$ defined as:

$$
\mathrm{C}_{\mathrm{m}}\left(\varphi_{1}\right)_{\mathrm{t}}=\mathrm{I}_{\mathrm{m} 1}\left(\mathrm{~s}_{1}, \mathrm{~s}_{2}\right)+\mathrm{I}_{\mathrm{m} 2}\left(\mathrm{~s}_{1}-\mathrm{s}_{1}, \mathrm{~s}_{2}-\mathrm{s}_{2}\right)-\mathrm{I}_{\text {ion }}
$$

The transmembrane ion currents per unit volume are $\mathrm{I}_{\mathrm{m} 1}, \mathrm{I}_{\mathrm{m} 2}$ :

$$
\begin{aligned}
& \mathrm{I}_{\mathrm{m} 1}\left(\mathrm{~s}_{1}, \mathrm{~s}_{2}\right)=\mathrm{M}_{\mathrm{vs}}\left\{\frac{-2\left(\mu_{\mathrm{s} 1}-\mu_{\mathrm{s} 2}\right)}{\left(1+\mu_{\mathrm{s} 1}\right)\left(1+\mu_{\mathrm{s} 2}\right)} \operatorname{arctg}\left(\frac{\mathrm{ds}_{2}}{\mathrm{ds}_{1}} \sqrt{\frac{\mathrm{Gs}_{2}}{\mathrm{Gs}_{1}}}\right)+\frac{\mathrm{g}_{\mathrm{os}_{2}}}{\mathrm{Gs}_{2}}\right\} \times \\
& \times\left(\left(\frac{\mathrm{g}_{\mathrm{os}_{1}}}{\varphi_{\mathrm{c}}}\left(\varphi_{1}\right) \mathrm{s}_{1}\right) \mathrm{s}_{1}+\left(\frac{\mathrm{g}_{\mathrm{os}}}{\varphi_{1}}\left(\varphi_{1}\right) \mathrm{s}_{2}\right) \mathrm{s}_{2}\right), \\
& \mathrm{I}_{\mathrm{m} 2}\left(\mathrm{~s}_{1}-\mathrm{s}_{1}, \mathrm{~s}_{2}-\mathrm{s}_{2}\right)=\mathrm{M}_{\mathrm{vs}} \iint_{\mathrm{x}} \frac{\mu_{\mathrm{s} 1}-\mu_{\mathrm{s} 2}}{2 \pi\left(1+\mu_{\mathrm{s}_{1}}\right)\left(1+\mu_{\mathrm{s}_{2}}\right) \mathrm{G}} \times \\
& \times \frac{\left(\mathrm{s}_{1}-\mathrm{s}_{1}\right)^{2} / \mathrm{G} \mu_{\mathrm{s}_{1}}-\left(\mathrm{s}_{2}-\mathrm{s}_{2}\right)^{2} / \mathrm{G} \mu_{\mathrm{s}_{2}}}{\left[\left(\mathrm{~s}_{1}-\mathrm{s}_{1}\right)^{2} / \mathrm{G}_{\mathrm{s}_{1}}+\left(\mathrm{s}_{2}-\mathrm{s}_{2}\right)^{2} / \mathrm{G}_{\mathrm{s}_{2}}\right]} \times \\
& \times\left(\left(\frac{\mathrm{g}_{\mathrm{os}_{1}}}{\lambda_{\mathrm{c}}}\left(\varphi_{1}\right) \mathrm{s}_{1}\right) \mathrm{s}_{1}+\left(\frac{\mathrm{g}_{\mathrm{os}_{2}}}{\lambda_{\mathrm{c}}}\left(\varphi_{1}\right) \mathrm{s}_{2}\right) \mathrm{s}_{2}\right) \mathrm{ds}_{1} \mathrm{ds}_{2} .
\end{aligned}
$$

here:

$$
\begin{gathered}
\mu_{s_{1}}=g_{o s_{1}} / g_{i s_{1}}, \mu_{s_{2}}=g_{\mathrm{os}_{2}} / g_{\mathrm{is}_{2}}, \\
G_{s_{1}}=\frac{g_{\mathrm{os}_{1}}+g_{\mathrm{is}_{1}}}{\lambda_{\mathrm{c}}}, \mathrm{G}_{\mathrm{s}_{2}}=\frac{\mathrm{g}_{\mathrm{os}_{2}}+\mathrm{g}_{\mathrm{is}_{2}}}{\lambda_{1}}, \mathrm{G}=\sqrt{\mathrm{G}_{\mathrm{s}_{1}} \mathrm{G}_{\mathrm{s}_{2}}} .
\end{gathered}
$$

The following notations are: $\mathrm{C}_{\mathrm{m}}$ - the capacitance of smooth muscle; $\mathrm{g}_{\mathrm{is} 1}, \mathrm{~g}_{\mathrm{is} 2}, \mathrm{~g}_{\mathrm{os} 1}, \mathrm{~g}_{\mathrm{os} 2}-$ the maximal intracellular (the subscript (i) and interstitial space), (the subscript (o) conductivity of the longitudinal and circular muscle layers in the longitudinal and circumferential directions), respectively; $\mathrm{M}_{\mathrm{vs}}$ - the membrane volume to surface ratio; $\mathrm{I}_{\text {ion }}$ - the total ionic current.

In the case of propagation along the isotropic circular muscle layer $\left(\varphi_{c}\right)$ :

$$
C_{m}\left(\varphi_{c}\right)_{t}=\frac{M_{v s}}{\left(1+\mu_{s_{1}}\right)}\left(\frac{g_{o s_{1}}}{\lambda_{c}}\left(\varphi_{1}\right)_{s_{1}}\right) s_{1}+\left(\frac{g_{o s_{2}}}{\lambda_{c}}\left(\varphi_{1}\right) s_{2}\right) s_{2}-I_{\text {ion }},
$$

where the above-mentioned abbreviations are used.

The points of the wall lie on the surface of the sphere throughout all stages of dynamic reaction.

$$
\left(\mathrm{Z}_{\mathrm{c}}-\mathrm{u}_{\mathrm{z}}\right)^{2}+\left(\mathrm{r}_{0}+\mathrm{u}_{\mathrm{r}}\right)^{2}+\left(\mathrm{r}_{0}+\mathrm{u}_{\mathrm{s}}\right)^{2}-\mathrm{R}^{2}=0, \mathrm{z} \in\left[\mathrm{z}_{1}, \mathrm{z}_{2}\right]
$$


Here: $u\left(u_{z}, u_{r}, u_{s}\right)$ - the displacement vector; $Z_{c}-$ the position of the center of the sphere at time $t ; z_{1}, z_{2}-$ the boundary points of contact of sphere and the wall. The kinematic equation of the motion of the bolus along the tube is governed by:

$$
\eta \frac{d Z_{c}}{d t}+F_{d}=2 \pi r_{0} \int_{z_{1}}^{z_{2}} \int_{r_{0}}^{r} F_{c} d \xi d \xi
$$

where $F_{c}, F_{d}$ are the contact force and the force of dry friction, respectively, $\eta$ is the coefficient of viscous friction.

At the initial moment of time, the whole system is in the resting state. It is supposed that the left and right boundaries of the tube are rigidly fixed. Discharges of the pacemaker cell trigger contractions in smooth muscle. When the maximum of the total force in the longitudinal muscle layer is achieved, contraction of the circular muscle layer starts. The complete activation of the circular muscle layer begins as the maximum of the total force in the longitudinal muscle layer is accomplished. The right boundary of the tubular segment remains in the resting state throughout.

Stages and sequences defining the dynamics of peristaltic propulsion of a solid sphere took part, as shown in Fig. 1. The calculations use a set of mechanical and electrical parameters and constants such as: $1=10.0 \mathrm{~cm}, \mathrm{r}_{0}=0.795 \mathrm{~cm} ; \gamma_{0}=26.14(\mathrm{~g} / \mathrm{cm} 2) ; \mathrm{c}_{71}=-40, \mathrm{c}_{81}=120, \mathrm{c}_{91}=-50$, $\mathrm{c}_{7 \mathrm{c}}=-25, \mathrm{c}_{8 \mathrm{c}}=75, \mathrm{c}_{9 \mathrm{c}}=-31.2(\mathrm{mN} / \mathrm{cm} \cdot \mathrm{mV}) ; \mathrm{k}=33.3(\mathrm{mN} \cdot \mathrm{sec} / \mathrm{cm}) ; \mathrm{p}_{\mathrm{o}}=1.0 \mathrm{kPa} ; \mathrm{C}_{\mathrm{m}}=1.0(\mu \mathrm{F} /$ $\left.\mathrm{cm}^{2}\right) ; \mathrm{M}_{\mathrm{vs}}=0.05$. The use of colon tissue prevented us from estimating some of the mechanical and electrical parameters used in the model. Therefore, the missing data have been adjusted during the numerical simulation: $\mathrm{c}_{1}=\mathrm{c}_{2}=10.4, \mathrm{c}_{3}=2.59, \mathrm{c}_{4}=3.79, \mathrm{c}_{5}=12.7, \mathrm{c}_{6}=0$; the conductivity constants in case of isotropy were: $\mathrm{g}_{\mathrm{is} 1}=2, \mathrm{~g}_{\mathrm{is} 2}=\mathrm{g}_{\mathrm{os} 1}=20, \mathrm{~g}_{\mathrm{os} 2}=80\left(\mathrm{mSm} / \mathrm{cm}^{2}\right)$; in a case of isotropy the conductivity constants were: $\mathrm{g}_{\text {is } 1}=2, \mathrm{~g}_{\mathrm{is} 2}=\mathrm{g}_{\mathrm{os} 1}=\mathrm{g}_{\mathrm{os} 2}=20\left(\mathrm{mSm} / \mathrm{cm}^{2}\right)$.

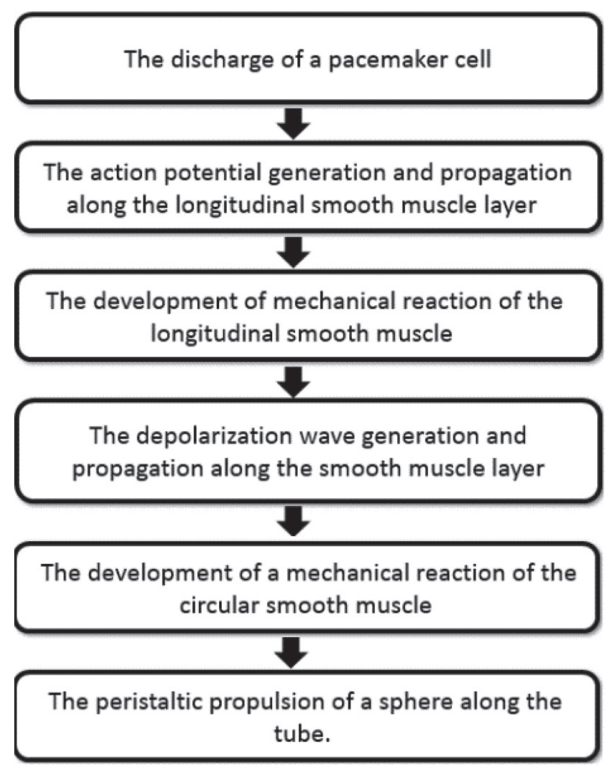

Figure 1: The sequence of events underlying the propulsion of a solid sphere. 


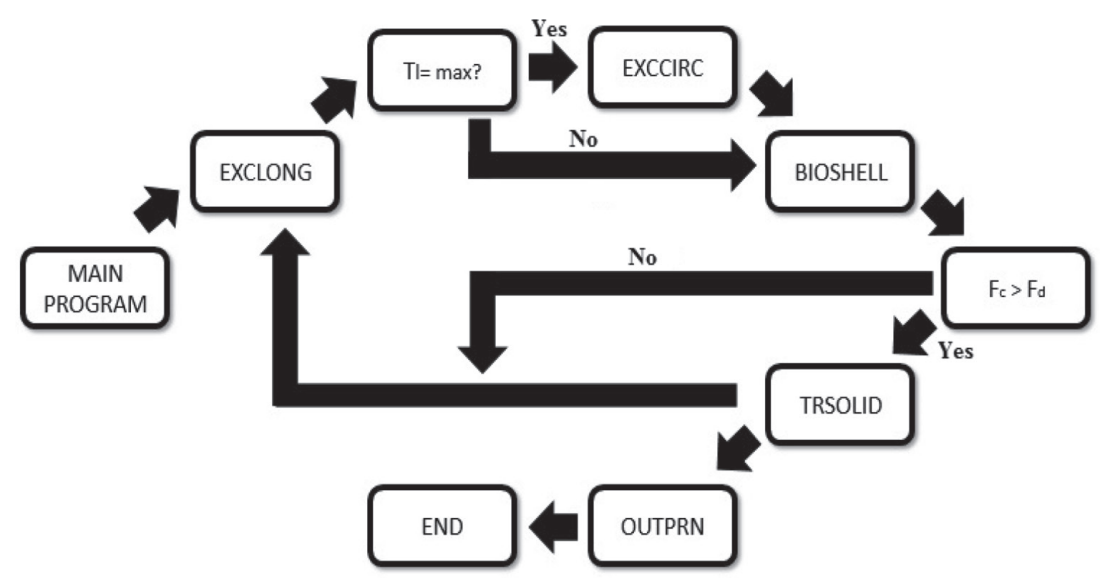

Figure 2: Flow chart of the algorithm.

Numerical algorithm flow chart is shown in Fig. 2. The main program is utilized to define the set of input parameters and constants, the initial configuration and the stress strain state of the tube. It includes the following subroutines:

EXCLONG (EXCCIRC) - Computes the dynamics of the depolarization wave long the longitudinal and circular smooth muscle bi-syncytia; uses the explicit finite-difference scheme of the second order approximation over the spinal and time variables.

BIOSHELL - Calculates the stress-strain states of the intestinal segment; uses the explicit hybrid finite-difference scheme of the second order approximation over the space and time. TRSOLID - Calculates the stress distribution over the enclosed sphere and its propulsion along the tube.

OUTPRN - Prints results.

\section{RESULTS}

The results of simulations of movement patterns resemble those recorded experimentally. They provide quantitative insights into the spatio-temporal patterns of changes in configuration, the distribution of contact forces over the bolus, and predict the average velocity of colonic transit. Therefore, a reciprocal relationship in the contraction of the longitudinal and circular smooth muscle is needed to guarantee the 'mixing' type of movements. Besides, strong interactive contractions of longitudinal and circular muscle layers are needed to expel the bolus from the gut. The dynamics of stress-strain distribution demonstrate the rise in the intensity of active propulsive forces in the circular smooth muscle layer throughout the entire phase of propulsion of the bolus. Viscous compared to dry friction had a marked effect on the average velocity of colon transit. Thus, the addition of osmotic and rapidly acting lubricant laxatives intraluminally shortened the time required for expulsion of the bolus in a significant way.

In the stimulation, $10 \mathrm{~cm}$ long of a part in the colon was constructed. The diameter of it is $0.795 \mathrm{~cm}$, bolus diameter is $1.5 \mathrm{~cm}$, viscous friction is $230 \mathrm{Ns} / \mathrm{m}$, dry friction is $20 \mathrm{~N}$, and intraluminal pressure is $0.1 \mathrm{kPa}$. Under these physiological circumstances, the bolus moves normally without any irritability within 2.4 seconds. However, whenever viscous friction increases, the irritability is increasing; hence the bolus takes long time to be expelled and as it decreases, the bolus will move normally and faster. 


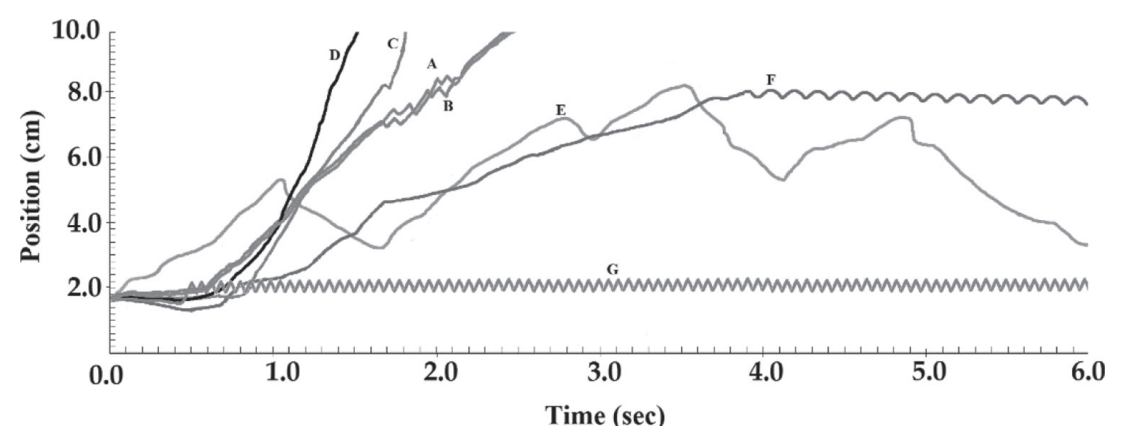

Figure 3: Movements of the bolus as a function of the viscous and dry friction. A: $\mu=230$ $\mathrm{Ns} / \mathrm{m} ; \mathrm{F}_{\mathrm{d}}=20 \mathrm{~N}$. B: $\mu=230 \mathrm{Ns} / \mathrm{m} ; \mathrm{F}_{\mathrm{d}}=2 \mathrm{~N} . \mathrm{C}: \mu=200 \mathrm{Ns} / \mathrm{m} ; \mathrm{F}_{\mathrm{d}}=20 \mathrm{~N} . \mathrm{D}: \mu=$ $90 \mathrm{Ns} / \mathrm{m} ; \mathrm{F}_{\mathrm{d}}=450 \mathrm{~N}$. E: $\mu=230 \mathrm{Ns} / \mathrm{m} ; \mathrm{F}_{\mathrm{d}}=450 \mathrm{~N} . \mathrm{F}: \mu=1200 \mathrm{Ns} / \mathrm{m} ; \mathrm{F}_{\mathrm{d}}=20 \mathrm{~N}$. G: $\mu=230 \mathrm{Ns} / \mathrm{m} ; \mathrm{F}_{\mathrm{d}}=800 \mathrm{~N}$.

Dry friction is a highly effective parameter. The bolus takes long time to be expelled by defecation if the parameter increases but it can be adjusted by decreasing the viscose friction. Hence, the bolus moves in special pattern if the dry friction is between $565 \mathrm{~N}$ and $2000 \mathrm{~N}$. On the other hand, the bolus moves faster if the dry friction decreases, as illustrated in Fig. 3.

Intraluminal pressure is an important parameter. As it increases or decreases, the bolus takes long time to be expelled despite the irritability. It can be stopped only if the intraluminal pressure becomes higher than $15 \mathrm{kPa}$, as shown in Fig. 4.

Increasing or decreasing the amount of calcium entering the slow and fast calcium channels and potassium - calcium channels will cause a disruption in the bowel movement that will lead to a delay in defecation, as described in Fig. 5.

If there are, no electrical pulses such as ICCs and the motor neurons reach the smooth muscles of the colon, the bolus moves due to the fine motor function of the mechanoreceptors, but this movement will be in an irregular pattern, which leads to a delay defecation. In addition, if there are no electrical impulses and mechanoreceptors, the bolus moves due to the elastic forces but in a slow manner and with disruption. This fault can be adjusted by decreasing the viscous friction, as it is shown in Fig. 6.

Finally, there is a strong relationship between diameter of the colon, diameter of the bolus and velocity. If the diameter of the bolus increases, the bolus moves very fast. On the other
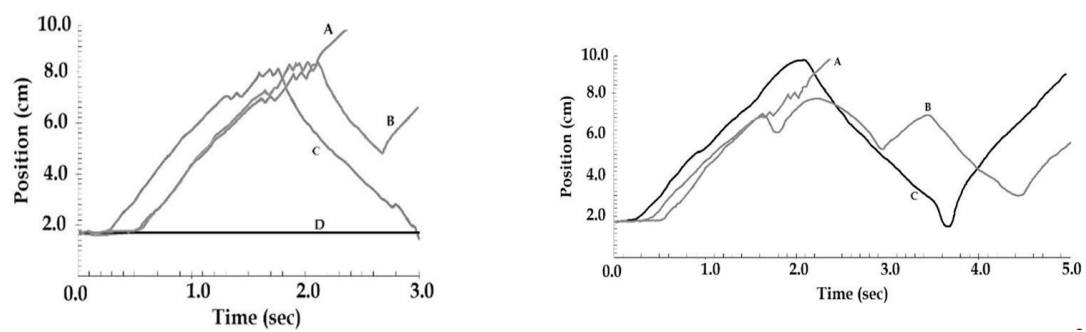

Figure 4: Effects of intraluminal pressure on propulsion. A: $p=0.1 \mathrm{kPa} . \mathrm{B}: \mathrm{p}=0.15 \mathrm{kPa}$. C: $\mathrm{p}=0.075 \mathrm{kPa} . \mathrm{D}: \mathrm{p}=20 \mathrm{kPa}$.

Figure 5: Effects of ion channel, $\mathrm{K}^{+}-\mathrm{Ca}^{2+}$, fast $\mathrm{Ca}^{2+}$, and slow $\mathrm{Ca}^{2+}$ permeability on bolus movements. A - norm; B - decreased permeability; C - increased permeability. 

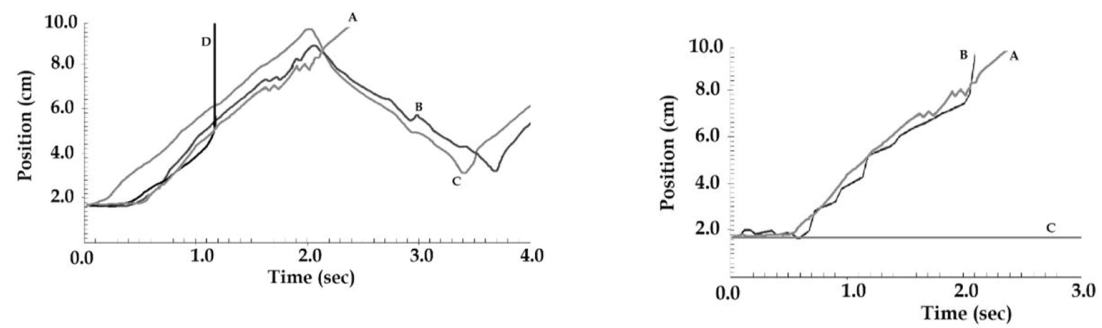

Figure 6: Effects of stimulation and viscous friction on bolus propulsion. A: $\mu=230 \mathrm{Ns} / \mathrm{m}$, with stimulation. B: $\mu=230 \mathrm{Ns} / \mathrm{m}$, no stimulation. C: $\mu=230 \mathrm{Ns} / \mathrm{m}$, with stimulation; D: $\mu=100 \mathrm{Ns} / \mathrm{m}$, no stimuli.

Figure 7: The effect of the radius on bolus movements. A: $r_{0}=0.795 \mathrm{~cm} . \mathrm{B}: \mathrm{r}_{0}=0.9 \mathrm{~cm}$. $\mathrm{C}: \mathrm{r}_{0}=0.6 \mathrm{~cm}$.

hand, if it decreases below $105 \%$ of the diameter of the colon, the bolus stops moving because mechanoreceptors will not fire and muscles of the colon cannot affect the bolus, as there are no stretching forces (Fig. 7).

\section{CONCLUSIONS}

The mathematical model of a segment of the gut reproduces qualitatively and quantitatively the dynamics of colonic transit. Viscous, and not dry, friction is the dominating parameter in the stability of propulsion. The reciprocal relationship of coordinated contractions of the longitudinal and circular smooth muscle layers is essential for the movement of the intraluminal content. Slow and fast calcium agonist/antagonist drugs must not be given to patients who suffer from constipation. Doctors should prescribe a combination of laxative lubricant drugs (mineral oil) and laxative bulk-formation (psyllium, methylcellulose) to have effective response.

\section{REFERENCES}

[1] Miftahof, R. \& Akhmadeev, N., Dynamics of intestinal propulsion. Journal of Theoretical Biology, 246, pp. 377-393, 2007. https://doi.org/10.1016/j.jtbi.2007.01.006

[2] Miftahof, R. \& Fedotov, E.M., Intestinal propulsion of a solid deformable bolus. Journal of Theoretical Biology, 235, pp. 57-70, 2005. https://doi.org/10.1016/j.jtbi.2004.12.019

[3] Miftahof, R. \& Abdusheva, G., Small bowel propulsion: Transit of a solid bolus. In Biophysics and Life Science, ed. D. Ghista. Munich: Springer, pp. 253-260, 2007. 